
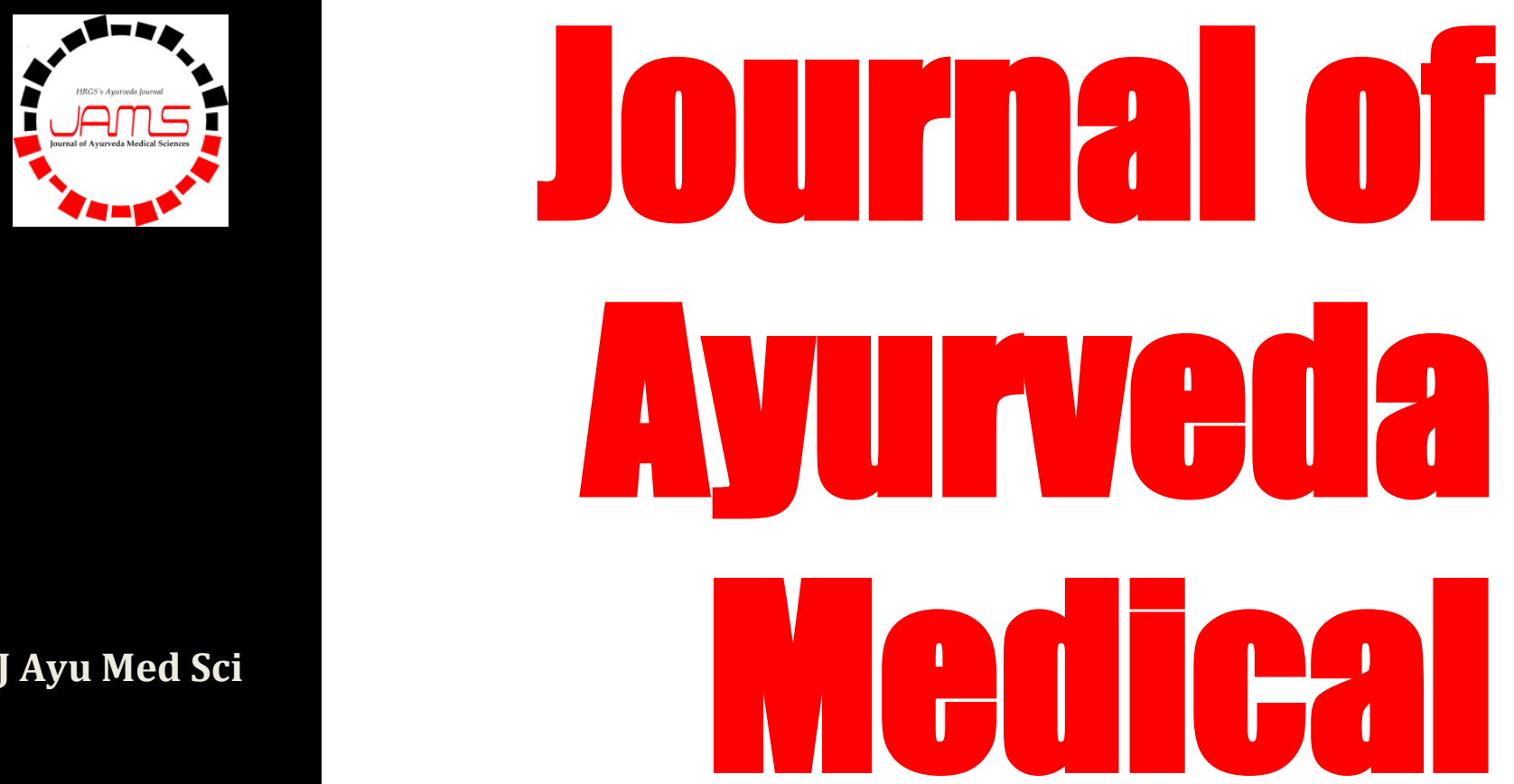

Quarterly Journal for

Rapid Publication

of Researches

in Ayurveda

and Other Traditional

Medicines

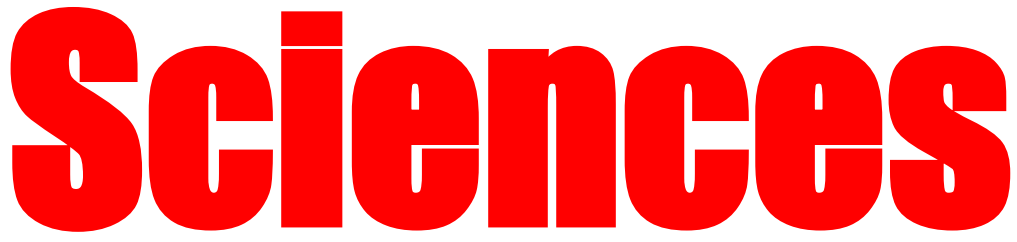

www.jayumedsci.com

ISSN 2456-4990

Chemical fingerprints for Panchavalkala Kvatha Curna

Panchavalkala Kwatha Curna (PKC) is an important poly-herbal formulation of Ayurveda used in the treatment of inflammation due to wound, ulcer, syphilis, leucorrhoea and conjunctivitis. Physico-chemical studies viz. total ash, water soluble ash, acid insoluble ash, water, alcohol and hydro - alcohol soluble extractive, loss on drying at $105^{\circ} \mathrm{C}, \mathrm{pH}, \mathrm{HPTLC}$ and LC-MS PKC and a monograph on quality standards for PKC is proposed from the data obtained to serve as a document to control the quality.

Koppala Narayana et al. 


\section{Chemical fingerprints for Panchavalkala Kvatha Curna}

Sunil Kumar Koppala Narayana ${ }^{1,2^{*}}$, Priyadarshini ${ }^{2}$, Puneeth ${ }^{2}$, Suchitra Narayana Prabhu ${ }^{2}$, Muralidhar Ballal ${ }^{3}$

${ }^{1}$ Current: Siddha Central Research Institute, CCRS, Ministry of AYUSH, Anna Hospital Campus, Arumbakkam, Chennai 600106. ${ }^{2}$ SDM Centre for Research in Ayurveda and Allied Sciences, ${ }^{3}$ SDM Ayurveda Pharmacy, Laxminarayana Nagar, Kuthpadi, Udupi 574118, India.

\section{ABSTRACT}

Introduction: Herbal medicines in recent times gained popularity and glory because of lesser known side effects/adverse effects and palatable and indispensable in its own way. Standardization as a benchmark to ascertain the quality, purity, safety and efficacy of the individual drug Panchavalkala kvatha curna (PKC) is a polyherbal formulation, compounded from parts of Nyagrodha (Ficus benghalensis stem bark), Udumbara (Ficus racemosa - stem bark), Asvattha (Ficus religiosa - stem bark), Parisa (Thespesia populnea - stem bark) and Plaksa (Ficus lacor - stem bark) as per formula composition in Ayurvedic Formulary of India. It is used in the treatment of inflammation due to wound, ulcer, syphilis, leucorrhoea and conjunctivitis. It can be used as lepa or in the form of decoction. In the current study chemical analysis and fingerprinting techniques have been employed to standardise PKC. Methods: PKC was subjected to organoleptic, macromicroscopic, physicochemical, HPTLC and LC-MS characterization employing standard methodology mentioned in pharmacopoeia and other herbal analysis protocols. Results: Physico-chemical studies viz. total ash, water soluble ash, acid insoluble ash, water, alcohol and hydro - alcohol soluble extractive, loss on drying at $105^{\circ} \mathrm{C}, \mathrm{pH}, \mathrm{HPTLC}$ and LCMS were performed. Conclusion: A monograph on quality standards for PKC is proposed from the data obtained. The results of the present investigation would serve as a document to control the quality of an important poly-herbal formulation of Ayurveda.

KEYWORDS HPTLC, LC-MS fingerprint, monograph, polyherba formulations, standardization.

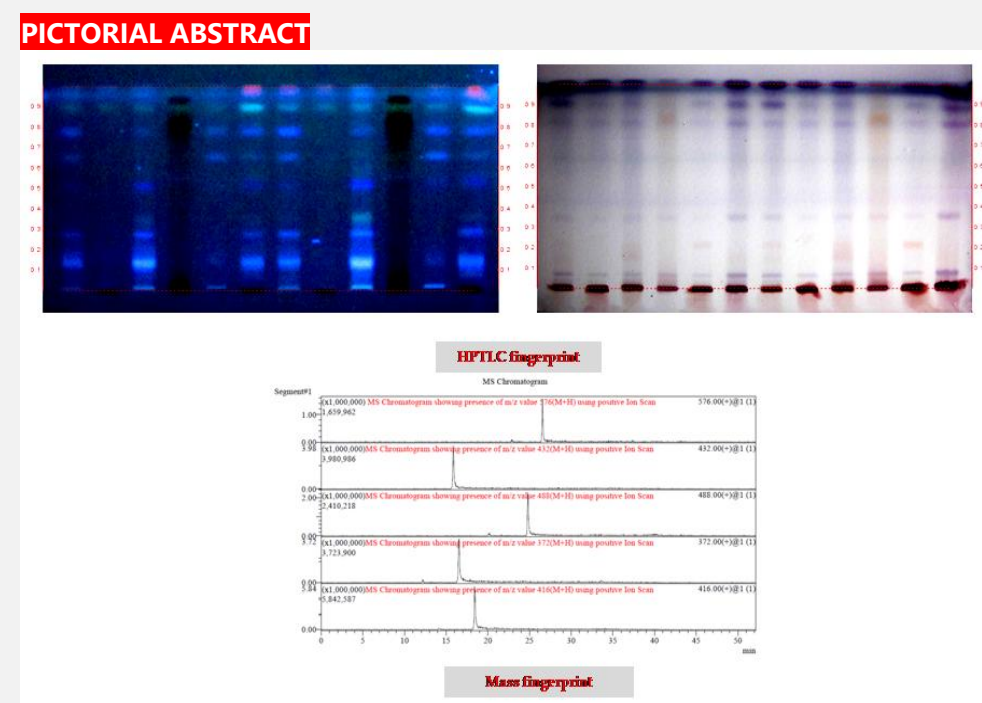

ARTICLE HISTORY Received 27.06.2018 Accepted 21.07.2018

CORRESPONDENCE Dr KN Sunil Kumar, Research Officer (Pharmacognosy), Siddha Central Research Institute, CCRS, Ministry of AYUSH, Anna Hospital Campus, Arumbakkam, Chennai 600106, India. Email: kn.sunil@gov.in

CITE THIS RESEARCH AS Koppala Narayana SK, Priyadarshini, Puneeth, Prabhu SN, Ballal M. Chemical fingerprints for Panchavalkala Kvātha Cūrṇa. J Ayu Med Sci2018;3(2):356-68.

DOI 10.5530/jams.2018.3.16

\section{INTRODUCTION}

Quality, safety and efficacy tests for obtaining standards

for Ayurvedic preparations are to be considered with due importance $^{[1]}$. Approval for quality standards, since authentication of botanical source of ingredients to preparation of finished product, each of the process has its own importance. Since there are several active principles in a single herbal drug ingredient standardisation of formulations with multiple crude drugs is a difficult task unlike modern drugs. Every herbal formulation in the Ayurvedic formulary needs standardisation employing all possible chemical means ${ }^{[2]}$.

Panchavalkala kvatha cūrna (PKC) is prescribed in conditions of inflammation due to wounds, syphilis, leucorrhoea, conjunctivitis as external therapyafter grinding with ghritha or as decoction. PKC is composed of coarse powder of stem barks of Nyagrodha (Ficus benghalensis L.), Udumbara (Ficus racemosa L.), Asvattha (Ficus religiosa L.), Parisa (Thespesia populnea (L.)
Sol. ex Correa) and Plaksa (Ficus lacor Buch.-Ham.) in equal proportions as mentioned in Ayurvedic Formulary of India $(\mathrm{AFI})^{[3]}$. The current study attempts to develop monograph on quality control parameters for PKC using physico - chemical, HPTLC and LCMS analytical tools.

\section{. MATERIALS AND METHODS}

\subsection{Collection and identification of plant samples}

Dry raw samples were collected from the raw drug section of SDM Ayurveda pharmacy, Udupi as well as some authentic raw material suppliers. The samples were authenticated using macro-microscopic examination, voucher specimens (No. SDM/RGU-MRP/PKC/01-05) have been deposited in the crude drug museum of Pharmacognosy department of SDMCRAAS, Udupi, Karnataka. 


\subsection{Method of Preparation of Panchavalkala kvātha cūrṇa}

There is no protocol for the preparation of kvātha cūrṇa (coarse powder) in Ayurvedic Pharmacopoeia of India (API) though there is protocol for preparation of cūrṇa (fine powder). PKC was prepared as per procedure detailed for cūrṇa in $\mathrm{API}^{[4]}$, with modification of sieve size (10 was used). All the ingredients of Pharmacopoeial quality were washed separately to have no microbial load ${ }^{[5]}$, dried, and coarsely powdered. The individual raw drug powders were passed separately through sieve number 10. Each ingredient was weighed separately and mixed together in equal proportions as per $\mathrm{AFI}^{[3]}$. The mixture was passed through sieve number 10 again to obtain a homogenous blend and packed in an air-tight container.

\subsection{Physico-chemical analysis}

Physico-chemical studies viz. total ash, water soluble ash, acid insoluble ash, water, alcohol and hydro - alcohol soluble extractive, loss on drying at $105^{\circ} \mathrm{C}$ and $\mathrm{pH}$ were carried out as per the standard procedures mentioned in $\mathrm{API}{ }^{[6]}$.

\subsection{TLC/HPTLC fingerprinting}

2.3.1 Ethanol extraction of ingredients and PKC

Five grams each of Nyagrodha, Udumbara, Asvattha, Parisa, Plaksa and PKC were extracted with $150 \mathrm{ml}$ of ethanol using Soxhlet apparatus. The filtrate was concentrated to dryness and $100 \mathrm{mg}$ of dried residue was dissolved in $5 \mathrm{ml}$ of solvent in a standard flask individually ${ }^{[7]}$.

2.3.2 Hydro-alcoholic extraction of PKC.

Five grams of PKC was extracted with ethanol and water (1:1) by maceration at room temperature for $24 \mathrm{hrs}$ with intermittent shaking followed by filtration and concentrating to 5 $\mathrm{ml}$.

\subsubsection{HPTLC}

2.3.3.1 Ethanol extract of PKC along with the extracts 5 ingredients were applied $(10 \mu \mathrm{l}$ each) on aluminium plates precoated with silica gel $60 \mathrm{~F}_{254}$ of $0.2 \mathrm{~mm}$ thickness using LINOMAT 5. The plate was developed in twin trough chamber previously saturated with mobile phase toluene: ethyl acetate: formic acid (5.0: 5.0: 0.2).

2.3.3.2 Hydro-alcoholic extract was applied to the concentrations of 4 and $8 \mu \mathrm{l}$ on aluminium plates pre-coated with silica gel $60 \mathrm{~F}$ ${ }_{254}$ of $0.2 \mathrm{~mm}$ thickness using LINOMAT 5 . The plate was developed in twin trough chamber previously saturated with mobile phase toluene: ethyl acetate: acetic acid: water (3.0: 3.0: $0.8: 0.2)$.

The developed plate was visualized in visualizing chamber and scanned in CAMAG TLC scanner 4 under 254, 366 (pre-derivatisation) and at $620 \mathrm{~nm}$ (post-derivatisation with vanillin - sulphuric acid (VSA)). With the help of CAMAG WinCATS software, $\mathrm{R}_{\mathrm{f}}$ values and densitograms were recorded ${ }^{[8,9]}$.

\subsection{LCMS fingerprinting}

In order to perform a qualitative analysis of the compounds present in PKC methanolic extract was analysed by LC/ESI/MS using Bruker UHPLC 3000 chromatography coupled to a quadrupole ToF mass selective detector (micrOTOF-QII). The operating conditions were as follows: column RP C18 (100 $\mathrm{mm} \times 3.9 \mathrm{~mm}$ ), internal diameter $=5 \mu \mathrm{m}$; elution gradient; mass spectra negative ion mode; mobile phase: $0.5 \mathrm{ml}$ of ortho phosphoric acid and $136 \mathrm{mg}$ of $\mathrm{KH}_{2} \mathrm{PO}_{4}$ dissolved in $900 \mathrm{ml}$ of HPLC grade water, made up to $1000 \mathrm{ml}$ filtered through $0.45 \mu$ membrane and degassed in sonicator for $5 \mathrm{~min}$ (Solvent $\mathrm{A}$ ), acetonitrile (Solvent B); flow rate $1.2 \mathrm{ml} / \mathrm{min}$; injection volume $25 \mathrm{mg} / 10 \mathrm{ml}$ methanol ${ }^{[10]}$.

\section{RESULTS AND DISCUSSION}

Standardisation parameters are set by considering the importance of each test viz loss on drying, total ash content, acid insoluble ash, alcohol soluble extractive value and water soluble extractive value to assure uniformity in manufacturing and considering efficacy and safety. Physico - chemical constants for all the ingredients and the preparation PKC was estimated (Table 1). The physico - chemical constants for all the ingredients used in the preparation of $\mathrm{PKC}$ as well the same for PKC will be useful in quality control lab in future.

HPTLC profile by photo-documentation (Figure 1), densitometry (Figure 2-5) and $\mathrm{R}_{\mathrm{f}}$ values (Table 2-4) has been generated for PKC along with the 5 ingredients. Hydro-alcoholic extract of PKC was also fingerprinted in a mobile phase suitable for that polarity (Table 5, Figure 6, 7). Fingerprint patterns were documented under short UV, long UV and after derivatisation with VSA.

Under short UV Nyagrodha, Udumbara, Asvattha and Plaksa showed no bands while Parisa showed 2 green bands and PKC showed 4 bands 2 of them corresponded to Parisa (Figure 1.1 and Table 2).

Under long UV Nyagrodha, Udumbara, Asvattha, Parisa, Plaksha and PKC showed 7, 2, 9, 6, 6, and 9 bands respectively (all in blue fluorescent colour). Nine bands occurred in $\mathrm{PKC}$, of them 8 were from 5 of ingredients used in the formulation. Band with $\mathrm{R}_{\mathrm{f}} 0.08$ was formed after compounding of the ingredients to PKC. Band with $\mathrm{R}_{\mathrm{f}} 0.14,0.66$ and 0.78 were observed in Nyagrodha, Asvattha and Plaksa. Band with $\mathrm{R}_{\mathrm{f}} 0.83$ and 0.94 (fluorescent black) were observed in Parisa. Band with $R_{f}$ 0.90 (F. Aqua) and 0.98 (F. Blue) was observed commonly in 
Nyagrodha, Udumbara, Asvattha, Parisa, and Plaksha (Figure 1.2 and Table 3).

After derivatisation with VSA, Nyagrodha, Udumbara, Asvattha, Parisa, Plaksha and PKC showed 8, 6, 8, 5, 6 and 5 bands respectively (of different colours). Out of five bands occurred in PKC, 3 were from 4 of ingredients used for the formulation, and 2 were from five ingredients used in PKC. Bands with $\mathrm{R}_{\mathrm{f}} 0.06,0.35$ (violet) were observed in all the five ingredients and bands with $R_{f}$ 0.81, 0.90, 0.99 (violet) were observed in Nyagrodha, Udumbara, Asvattha and Plaksha (Figure 1.3 and Table 4).

Densitometric scan of PKC showed 5, 5 and 8 peaks at 254, 366 and $540 \mathrm{~nm}$ after derivatisation with VSA (Figure 2.6, $3.6,4.6)$.
HPTLC performed for hydro-alcoholic extract of PKC at short UV showed 4 bands (green) at long UV showed 11 bands (fluorescent light violet) and after derivatisation (white light) showed 6 bands (light violet) respectively. Densitometric scan carried out showed 6 peaks at 254nm, 4 peaks at $366 \mathrm{~nm}$ and 4 peaks at $620 \mathrm{~nm}$ (after derivatisation with VSA) (Table 5).

Methanolic extract of PKC along with its 5 ingredients were analysed using LCMS for qualitative fingerprint purpose (Figure 8-9). The fingerprint has shown peaks from each extract.

Table 1. Raw data of physico-chemical constants of raw materials used in Panchavalkala kvatha curna

\begin{tabular}{|c|c|c|c|c|c|c|}
\hline Sample & LOD & TA & AIA & ASE & WSE & $p \mathrm{H}$ \\
\hline Nyagrodha & $8.13 \pm 0.30$ & $4.99 \pm 0.05$ & $0.45 \pm 0.05$ & $6.26 \pm 0.40$ & $9.28 \pm 0.14$ & - \\
\hline Udumbara & $11.04 \pm 0.03$ & $17.04 \pm 0.05$ & $0.30 \pm 0.00$ & $11.93 \pm 0.20$ & $10.26 \pm 0.54$ & - \\
\hline Asvattha & $8.16 \pm 0.03$ & $7.90 \pm 0.18$ & $0.65 \pm 0.145$ & $8.96 \pm 0.325$ & $10.25 \pm 0.38$ & - \\
\hline Parisa & $7.54 \pm 0.34$ & $12.31 \pm 0.08$ & $1.35 \pm 0.05$ & $7.67 \pm 0.47$ & $8.51 \pm 1.03$ & - \\
\hline Plaksa & $6.24 \pm 0.06$ & $8.84 \pm 0.04$ & $1.10 \pm 0.10$ & $15.95 \pm 0.00$ & $9.52 \pm 0.01$ & - \\
\hline PKC & $7.68 \pm 0.05$ & $9.83 \pm 0.0$ & $0.60 \pm 0.0$ & $15.95 \pm 0.0$ & $7.44 \pm 0.18$ & $6.0 \pm 0.0$ \\
\hline
\end{tabular}

Results expressed as average \pm SEM of $\%$ w/w. LOD - Loss on drying at 105\% FM - Foreign matter; TA - Total ash; AIA - Acid Insoluble ash; ASE - Alcohol soluble extractive; WSE - Water soluble extractive. PKC - Panchaalkala kvatha chrna

Figure 1. TLC photo documentation of Ethanolic extract of Panchavalkala Kvatha Curna with Ingredients

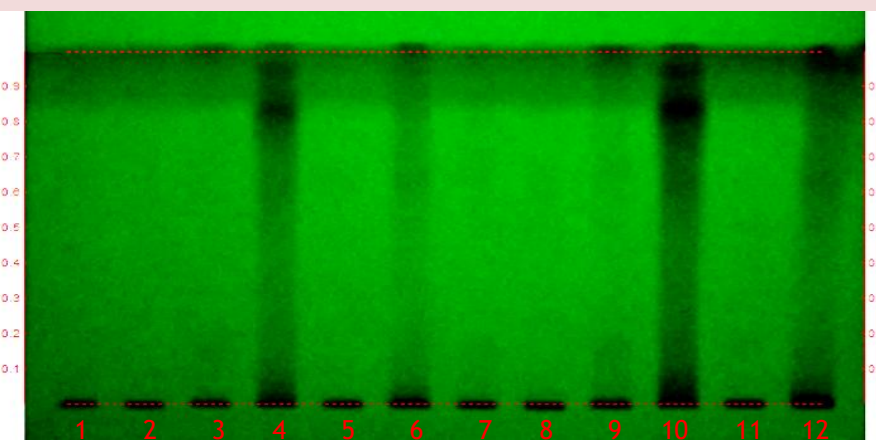

1.1 Under short UV

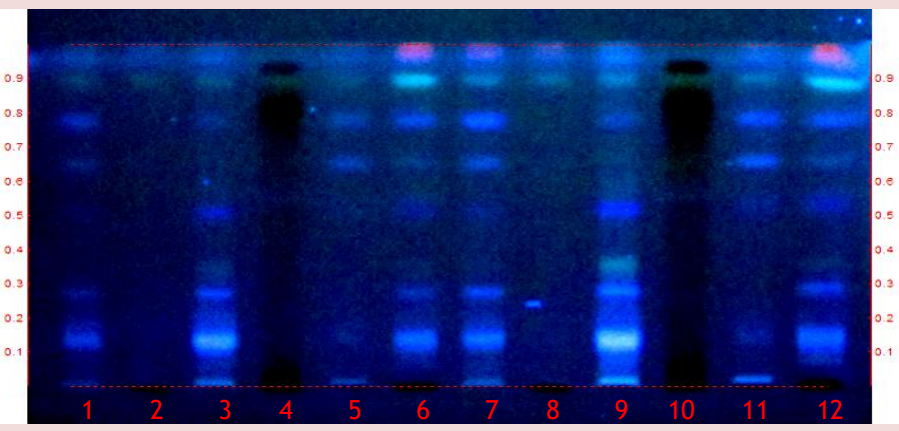

1.2 Under long UV 


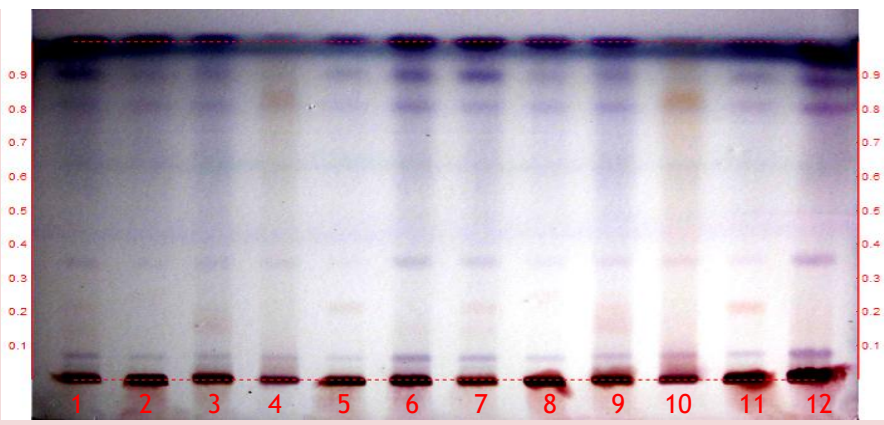

1.3 Under white light post derivatisation

Solvent system - Toluene: Ethyl Acetate: Formic Acid (5:5:0.2)

Track 1 - Nyagrodha (Ficus benghalensis); Track 2 - Udumbara (Ficus racemosa); Track 3 - Asvattha (Ficus religiosa); Track 4 - Parisa (Thespesia populnea); Track 5 - Plaksa (Ficus lacor); Track 6 - Panchavalkala Kvatha Curna (PKC)

Table 2. $\mathrm{R}_{\mathrm{f}}$ values of ingredients and Panchavalkala Kvatha Curna under short UV

\begin{tabular}{|c|c|c|c|c|c|}
\hline Nyagrodha & Udumbara & Asvattha & Parisa & Plaksa & PKC \\
\hline- & - & - & - & - & 0.64 L Green \\
\hline- & - & - & 0.84 D Green & - & 0.84 L Green \\
\hline- & - & - & 0.95 D Green & - & 0.95 L Green \\
\hline \multicolumn{5}{c}{} \\
\hline \multicolumn{5}{c}{ D - Dark; L - Light } \\
\hline
\end{tabular}

Table 3. $\mathrm{R}_{\mathrm{f}}$ values of ingredients and Panchavalkala Kvatha Curna under long UV

\begin{tabular}{|c|c|c|c|c|c|}
\hline Nyagrodha & Udumbara & Asvattha & Parisa & Plaksa & PKC \\
\hline - & - & - & - & - & 0.08 F L Blue \\
\hline 0.14 F Blue & - & 0.14 F Blue & - & 0.14 F L Blue & 0.14 F Blue \\
\hline 0.28 F Blue & - & 0.28 F Blue & - & - & 0.28 F Blue \\
\hline - & - & $0.36 \mathrm{~F}$ Aqua & - & - & $0.36 \mathrm{~F}$ Aqua \\
\hline 0.51 F L Blue & - & 0.51 F D Blue & - & - & - \\
\hline - & - & - & 0.54 F L Blue & 0.54 F Blue & 0.54 F Blue \\
\hline - & - & 0.60 F L Green & - & - & - \\
\hline 0.66 F L Blue & - & 0.66 F L Green & 0.66 F L Green & 0.66 F Blue & 0.66 F L Blue \\
\hline 0.78 F Blue & - & 0.78 F L Blue & - & 0.78 F Blue & $0.78 \mathrm{~F}$ Blue \\
\hline - & - & - & 0.83 F Black & - & - \\
\hline $0.90 \mathrm{~F}$ Aqua & 0.90 F Aqua & $0.90 \mathrm{~F}$ Aqua & 0.90 F Aqua & 0.90 F Aqua & $0.90 \mathrm{~F}$ aqua \\
\hline - & - & - & 0.94 F Black & - & - \\
\hline 0.98 F L Pink & 0.98 F L Pink & 0.98 F L Blue & 0.98 F L Blue & 0.98 F L Pink & 0.98 F Red \\
\hline
\end{tabular}


Table 4. $\mathrm{R}_{\mathrm{f}}$ values of ingredients and Panchavalkala Kvatha Curna under white light after derivatisation

\begin{tabular}{|c|c|c|c|c|c|}
\hline Nyagrodha & Udumbara & Asvattha & Parisa & Plaksa & PKC \\
\hline 0.06 L Violet & 0.06 L Violet & 0.06 L Violet & 0.06 L Violet & 0.06 L Violet & 0.06 Violet \\
\hline 0.14 L Brown & - & - & - & - & - \\
\hline- & - & 0.16 L Brown & - & - & - \\
\hline 0.22 L Brown & - & 0.22 L Brown & - & 0.22 L Brown & - \\
\hline- & 0.25 L Brown & - & - & - & - \\
\hline 0.35 L Violet & 0.35 L Violet & 0.35 L Violet & 0.35 L Violet & 0.35 L Violet & 0.35 Violet \\
\hline 0.42 L Violet & - & 0.42 L Violet & 0.42 L Brown & - & - \\
\hline 0.81 Violet & 0.81 Violet & 0.81 Violet & - & 0.81 L Violet & 0.81 Violet \\
\hline - & - & - & 0.83 L Brown & - & - \\
\hline 0.90 Violet & 0.90 Violet & 0.90 Violet & - & 0.90 L Violet & 0.90 Violet \\
\hline - & - & - & 0.95 L Brown & - & - \\
\hline
\end{tabular}

Figure 2. Densitometric scan of ingredients and Panchavalkala Kvatha at $254 \mathrm{~nm}$

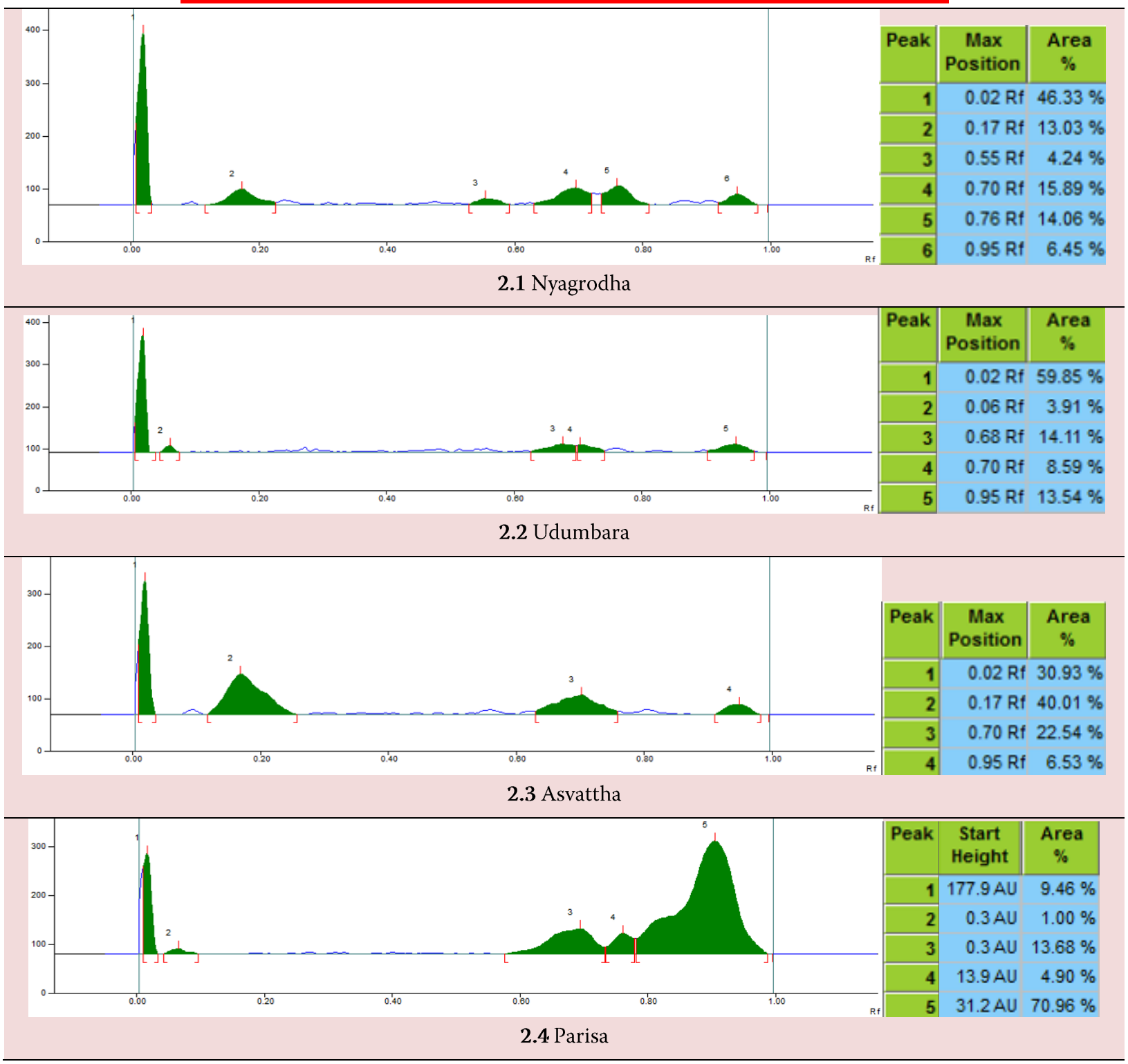




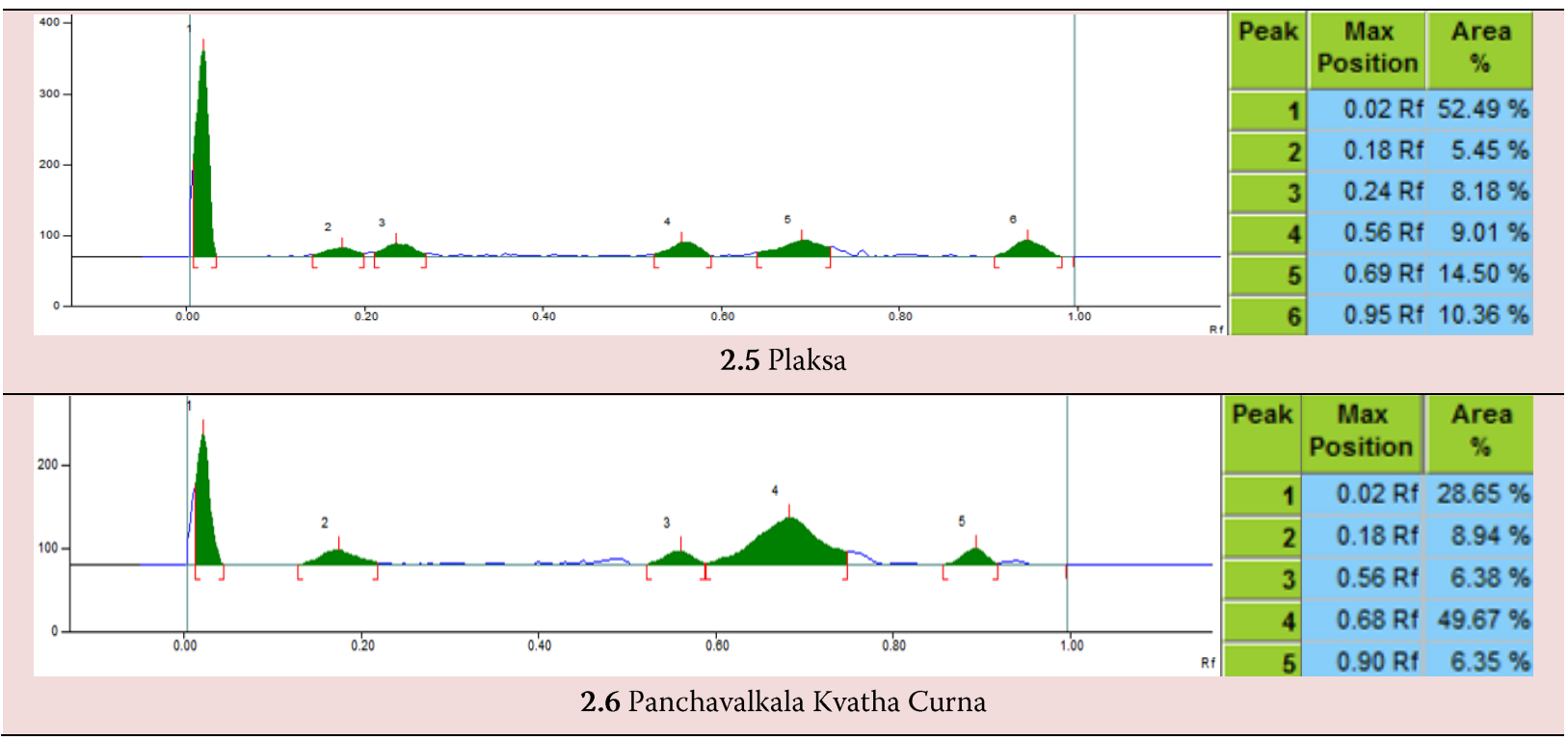

Figure 3. Densitometric scan of ingredients and Panchavalkala Kvatha at $366 \mathrm{~nm}$

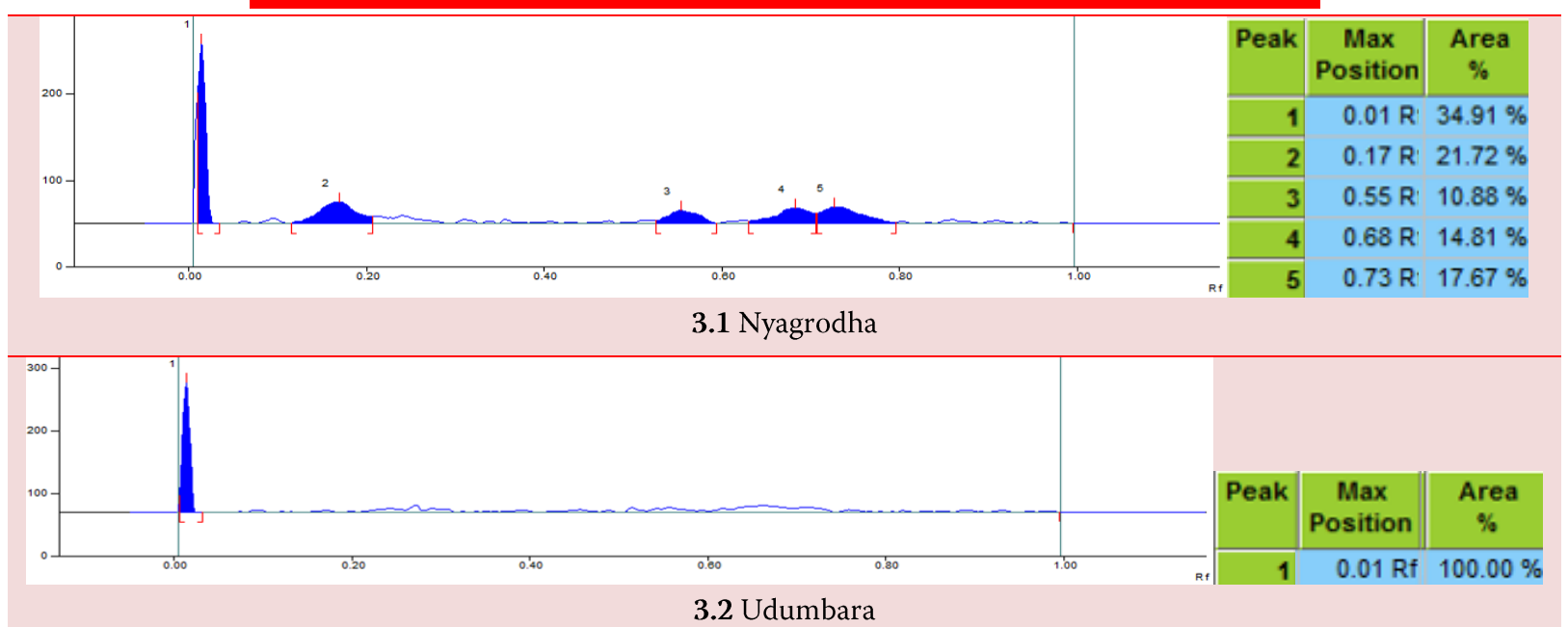

3.2 Udumbara
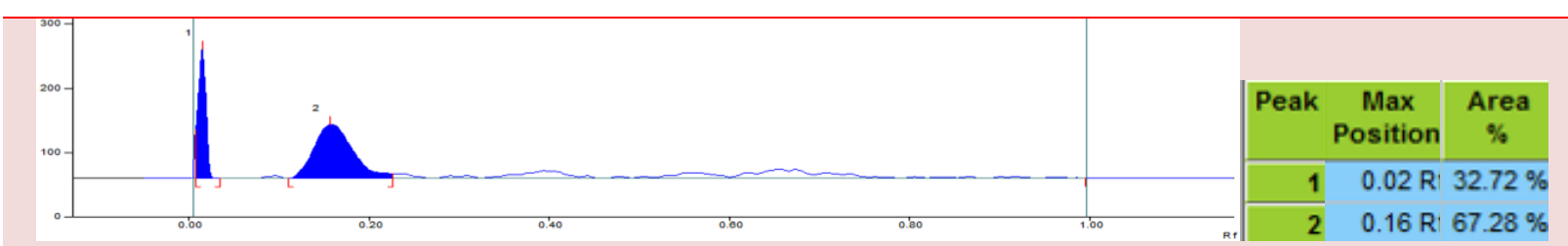

3.3 Asvattha
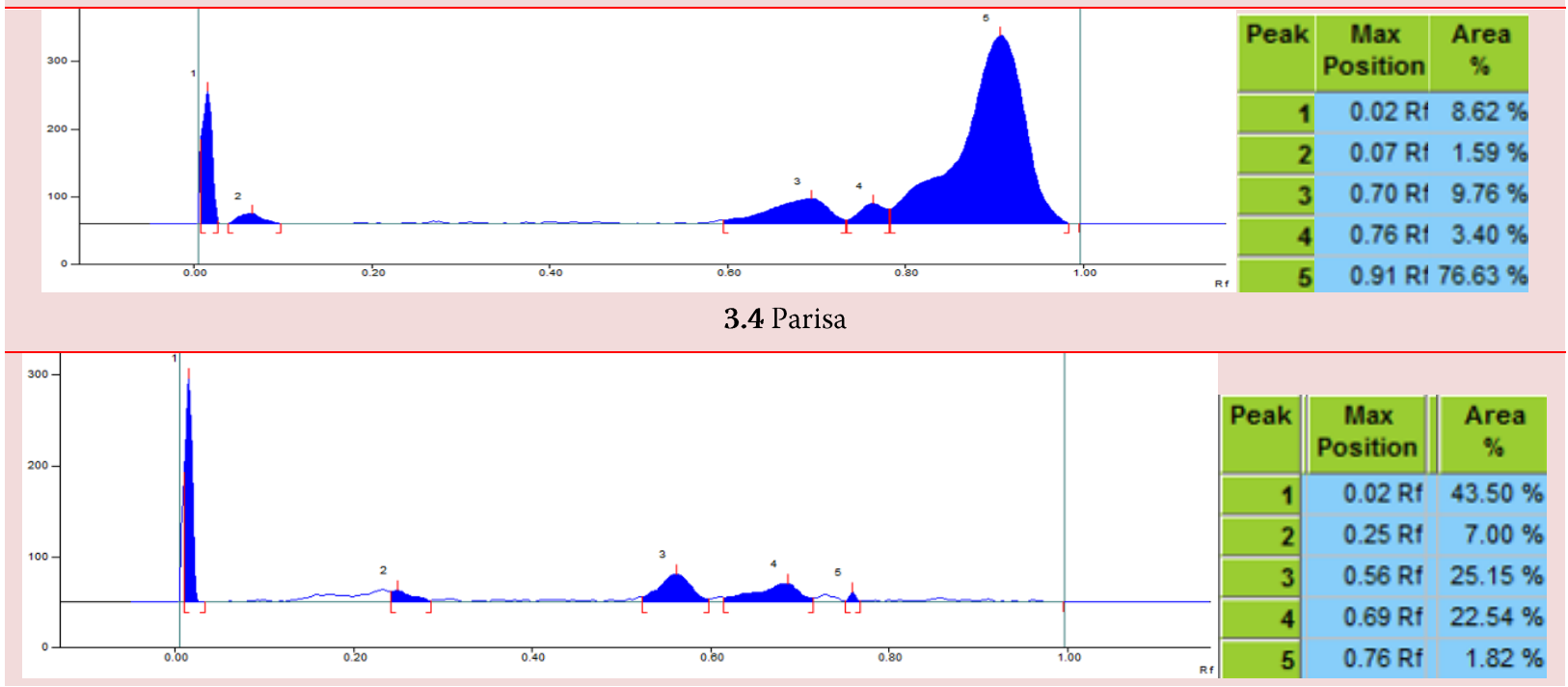


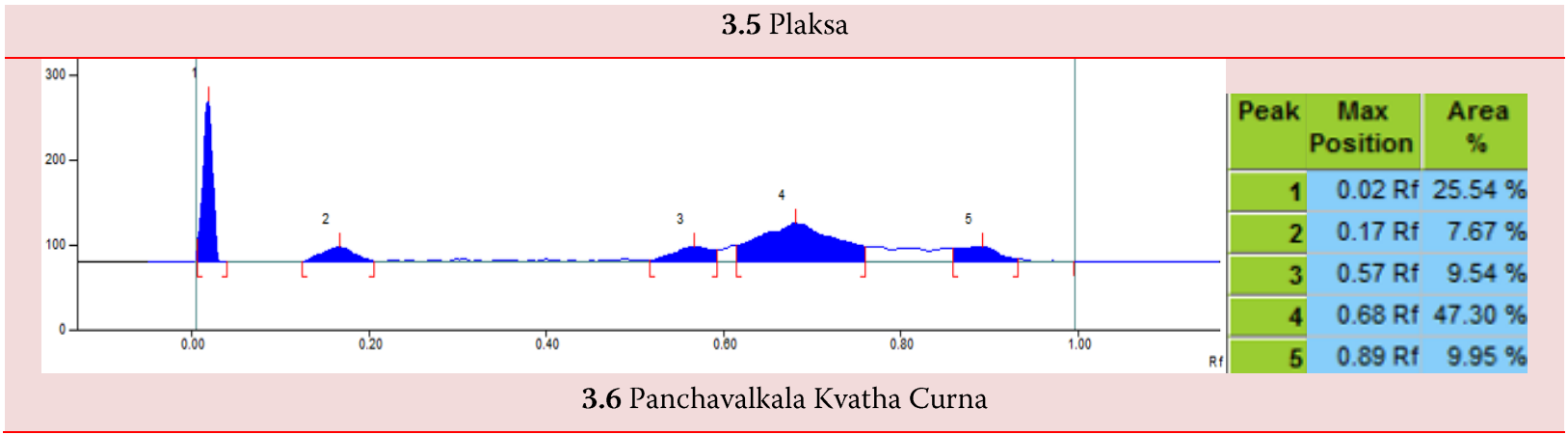

Figure 4. Densitometric scan of ingredients and Panchavalkala Kvatha at $620 \mathrm{~nm}$ after derivatisation $(10 \mu \mathrm{l})$

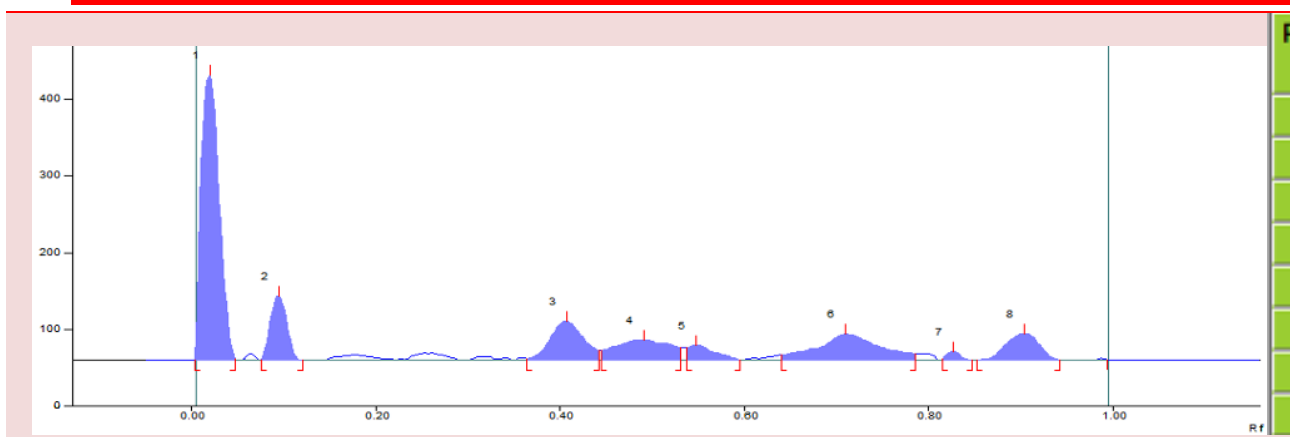

\begin{tabular}{|r|r|r|}
\hline Peak & $\begin{array}{c}\text { Max } \\
\text { Position }\end{array}$ & \multicolumn{1}{c|}{$\begin{array}{c}\text { Area } \\
\%\end{array}$} \\
\hline $\mathbf{1}$ & $0.02 \mathrm{Rf}$ & $44.42 \%$ \\
\hline $\mathbf{2}$ & $0.10 \mathrm{Rf}$ & $9.01 \%$ \\
\hline $\mathbf{3}$ & $0.41 \mathrm{Rf}$ & $11.13 \%$ \\
\hline $\mathbf{4}$ & $0.49 \mathrm{Rf}$ & $9.67 \%$ \\
\hline $\mathbf{5}$ & $0.55 \mathrm{Rf}$ & $3.39 \%$ \\
\hline $\mathbf{6}$ & $0.71 \mathrm{Rf}$ & $13.92 \%$ \\
\hline $\mathbf{7}$ & $0.83 \mathrm{Rf}$ & $0.97 \%$ \\
\hline $\mathbf{8}$ & $0.90 \mathrm{Rf}$ & $\mathbf{7 . 4 8} \%$ \\
\hline
\end{tabular}

4.1 Nyagrodha

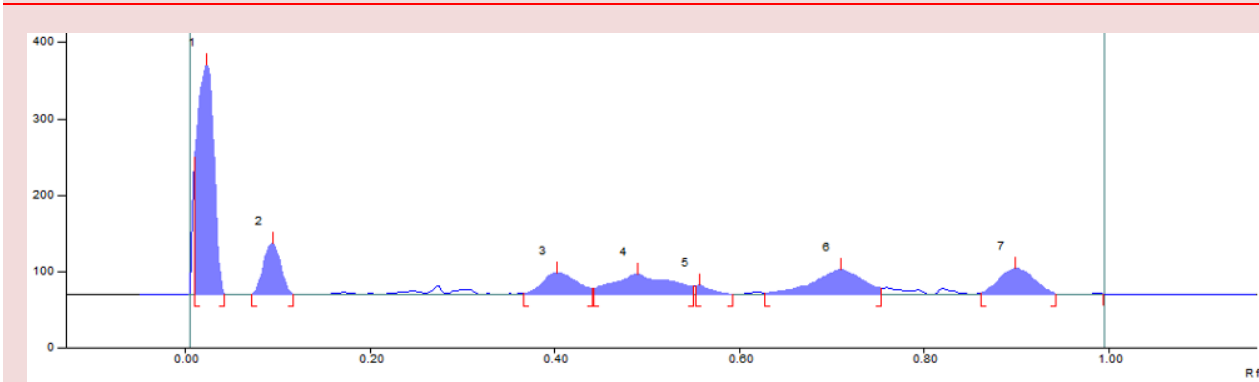

\begin{tabular}{|c|c|c|}
\hline Peak & $\begin{array}{c}\text { Max } \\
\text { Position }\end{array}$ & $\begin{array}{c}\text { Area } \\
\%\end{array}$ \\
\hline 1 & $0.02 R f$ & $42.81 \%$ \\
\hline 2 & $0.10 \mathrm{Rf}$ & $9.93 \%$ \\
\hline 3 & $0.40 \mathrm{Rf}$ & $8.35 \%$ \\
\hline 4 & $0.49 \mathrm{Rf}$ & $13.11 \%$ \\
\hline 5 & $0.56 \mathrm{Rf}$ & $1.63 \%$ \\
\hline 6 & $0.71 \mathrm{Rf}$ & $13.88 \%$ \\
\hline 7 & $0.90 \mathrm{Rf}$ & $10.29 \%$ \\
\hline
\end{tabular}

4.2 Udumbara

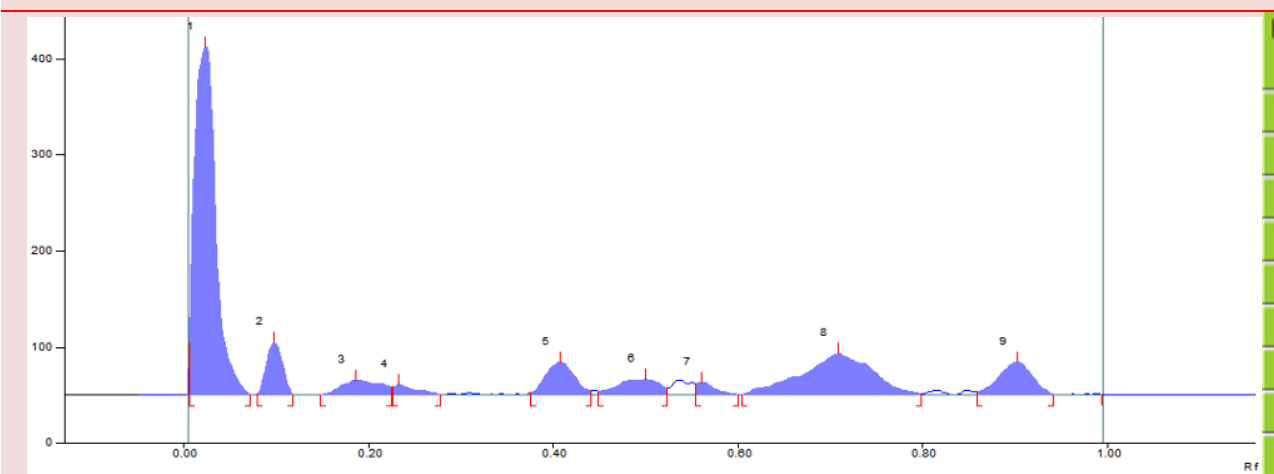

\begin{tabular}{|r|c|r|}
\hline Peak & $\begin{array}{c}\text { Max } \\
\text { Position }\end{array}$ & \multicolumn{1}{c|}{$\begin{array}{c}\text { Area } \\
\%\end{array}$} \\
\hline $\mathbf{1}$ & $0.02 \mathrm{Rf}$ & $49.98 \%$ \\
\hline $\mathbf{2}$ & $0.10 \mathrm{Rf}$ & $5.64 \%$ \\
\hline $\mathbf{3}$ & $0.19 \mathrm{Rf}$ & $3.90 \%$ \\
\hline $\mathbf{4}$ & $0.23 \mathrm{Rf}$ & $1.48 \%$ \\
\hline $\mathbf{5}$ & $0.41 \mathrm{Rf}$ & $6.30 \%$ \\
\hline $\mathbf{6}$ & $0.50 \mathrm{Rf}$ & $4.41 \%$ \\
\hline $\mathbf{7}$ & $0.56 \mathrm{Rf}$ & $1.62 \%$ \\
\hline $\mathbf{8}$ & $0.71 \mathrm{Rf}$ & $19.33 \%$ \\
\hline $\mathbf{9}$ & $0.90 \mathrm{Rf}$ & $7.35 \%$ \\
\hline
\end{tabular}

4.3 Asvattha

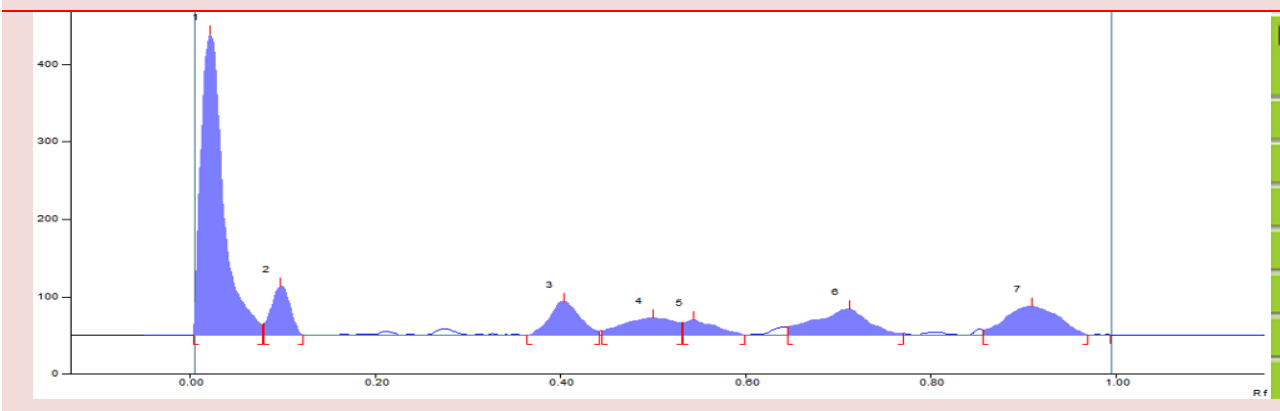

\begin{tabular}{|r|c|c|} 
Peak & $\begin{array}{c}\text { Max } \\
\text { Position }\end{array}$ & $\begin{array}{c}\text { Area } \\
\%\end{array}$ \\
\hline $\mathbf{1}$ & $0.02 \mathrm{Rf}$ & $52.06 \%$ \\
\hline $\mathbf{2}$ & $0.10 \mathrm{Rf}$ & $7.05 \%$ \\
\hline $\mathbf{3}$ & $0.40 \mathrm{Rf}$ & $\mathbf{7 . 7 8} \%$ \\
\hline $\mathbf{4}$ & $0.50 \mathrm{Rf}$ & $\mathbf{7 . 0 5} \%$ \\
\hline $\mathbf{5}$ & $0.55 \mathrm{Rf}$ & $3.57 \%$ \\
\hline $\mathbf{6}$ & $0.71 \mathrm{Rf}$ & $10.72 \%$ \\
\hline $\mathbf{7}$ & $0.91 \mathrm{Rf}$ & $11.76 \%$ \\
\hline
\end{tabular}

4.4. Parisa 

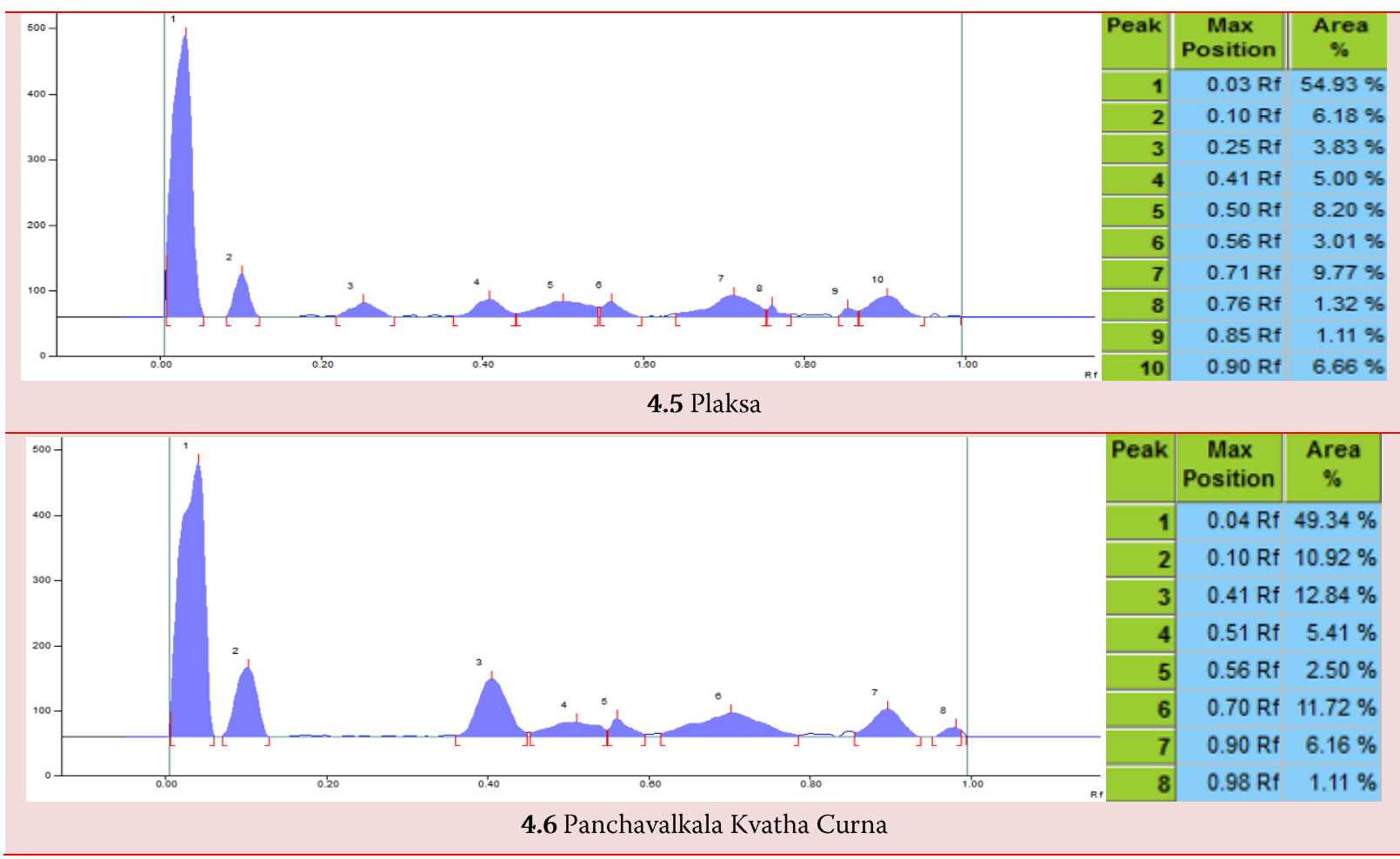

Figure 5. 3D display of ingredients and Panchavalkala Kvatha

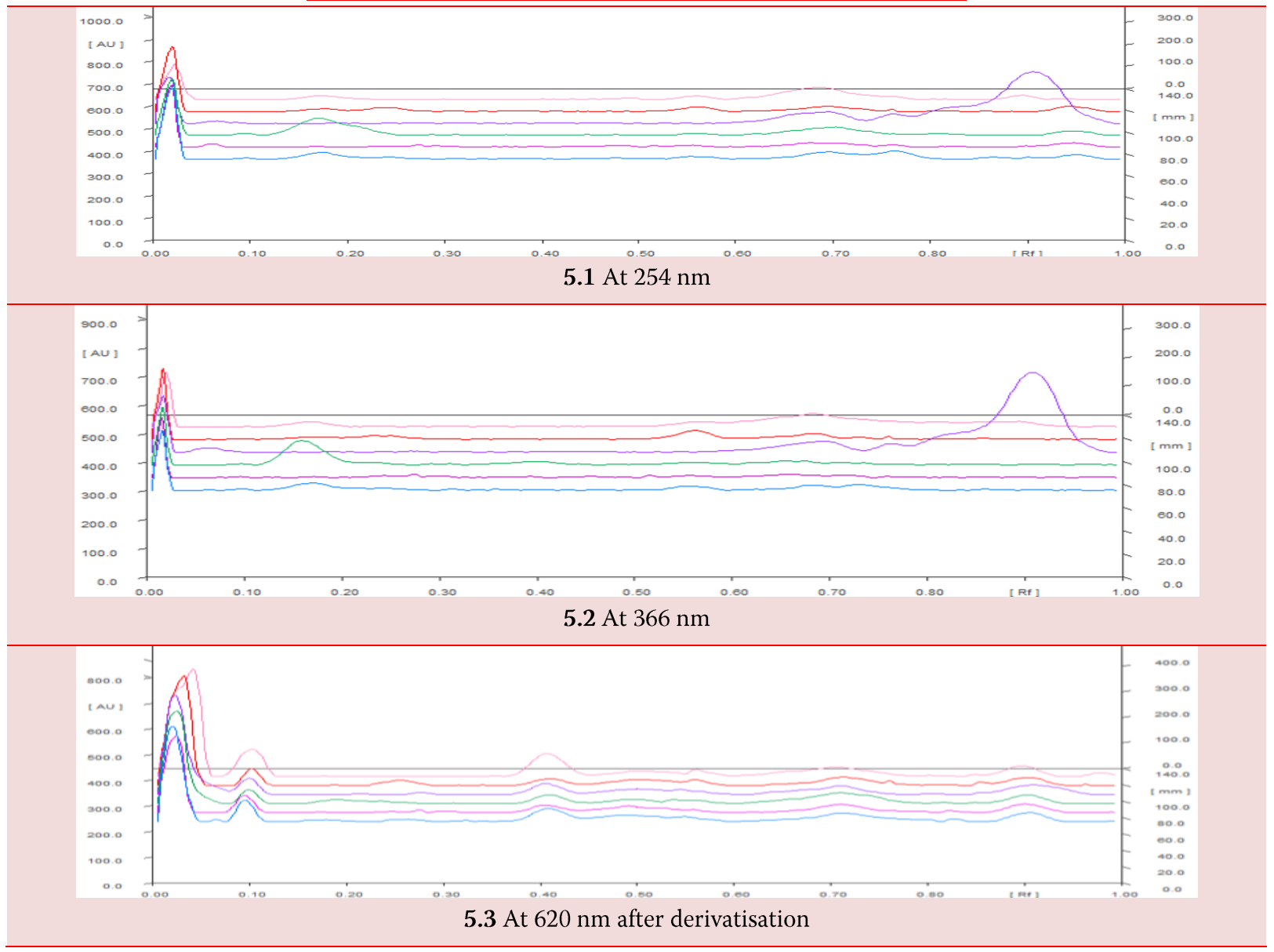




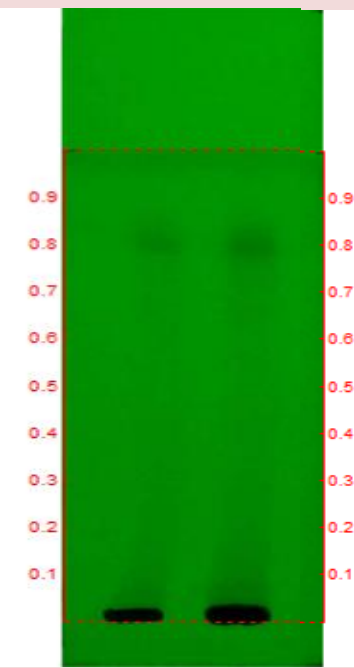

At $254 \mathrm{~nm}$

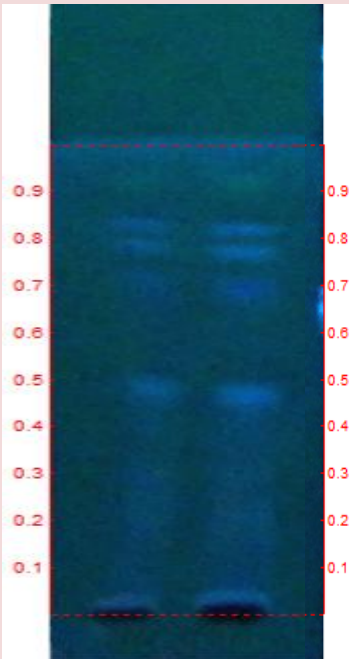

At $366 \mathrm{~nm}$

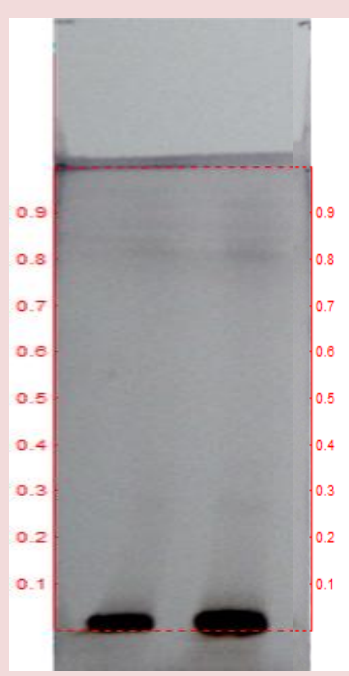

After Derivatisation

Solvent system: Toluene: Ethyl Acetate: Acetic acid: Water (3:3:0.8:0.2) Track 1-PKC- $4 \mu \mathrm{l}$; Track $2-\mathrm{PKC}-8 \mu \mathrm{l}$

\begin{tabular}{|c|c|c|}
\hline Under short UV & Under long UV & Under white light after derivatisation \\
\hline- & 0.05 F L Violet & - \\
\hline 0.07 L Green) & - & 0.07 L Violet \\
\hline- & 0.15 F L Violet & - \\
\hline- & $0.21 \mathrm{~F} \mathrm{~L} \mathrm{Violet}$ & - \\
\hline- & - & 0.27 L Violet \\
\hline- & 0.30 F L Violet & - \\
\hline- & 0.34 F L Violet & - \\
\hline- & 0.48 F Violet & - \\
\hline 0.67 L Green & - & - \\
\hline- & $0.69 \mathrm{~F}$ L Violet & - \\
\hline 0.71 L Green & $0.71 \mathrm{~F}$ L Violet & - \\
\hline- & 0.77 F L Violet & - \\
\hline $0.80 \mathrm{~L}$ Green & - & - \\
\hline- & - & $0.82 \mathrm{~L}$ Violet \\
\hline- & 0.84 F L Violet & 0.84 L Violet \\
\hline- & - & 0.87 L Violet \\
\hline- & 0.93 F L Green & 0.93 L Violet \\
\hline
\end{tabular}

L-Light, F-Fluorescence

Figure 7. Densitometric scan of hydro-alcoholic extract of Panchavalkadi Kwatha Curna

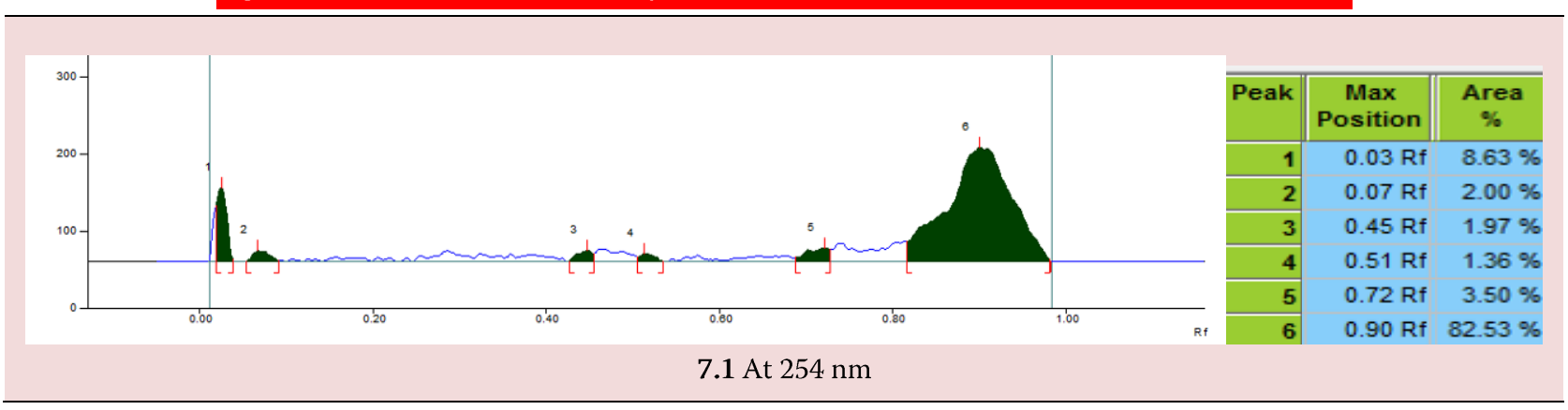




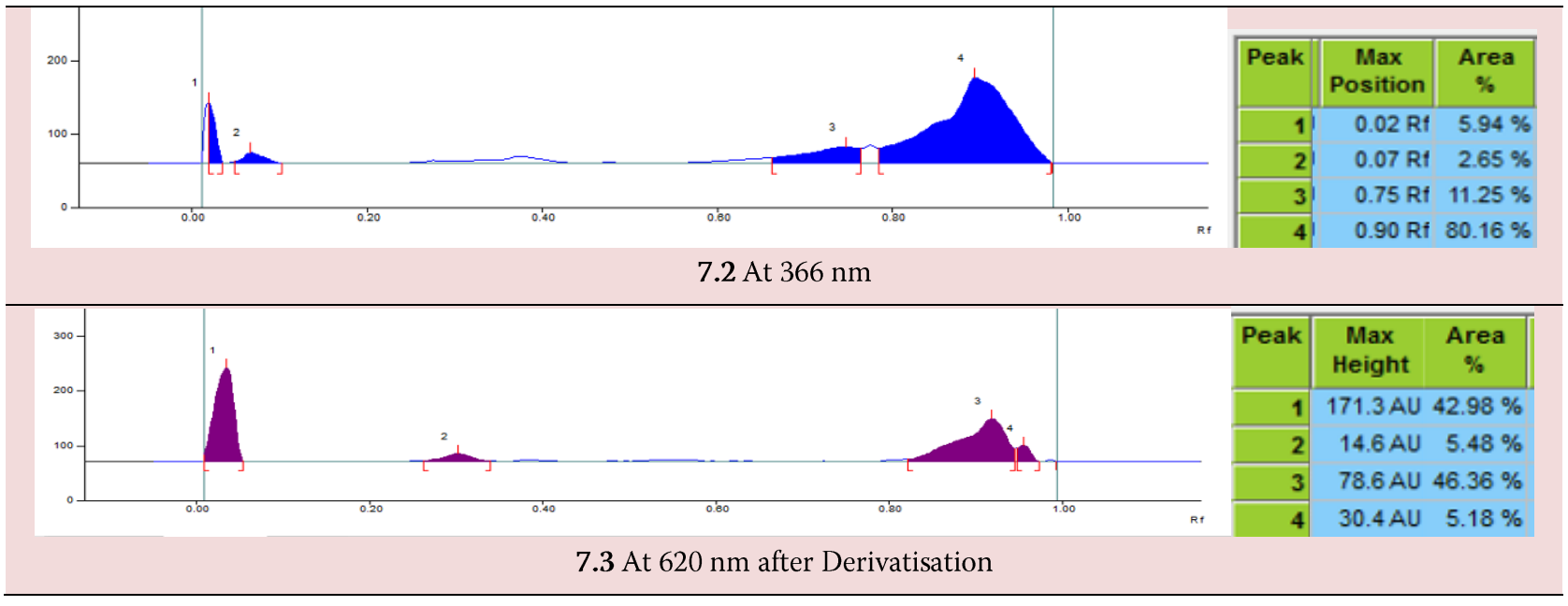

Figure 8. LCMS Fingerprint of methanolic extracts of ingredients of Panchavalakala kvatha curna
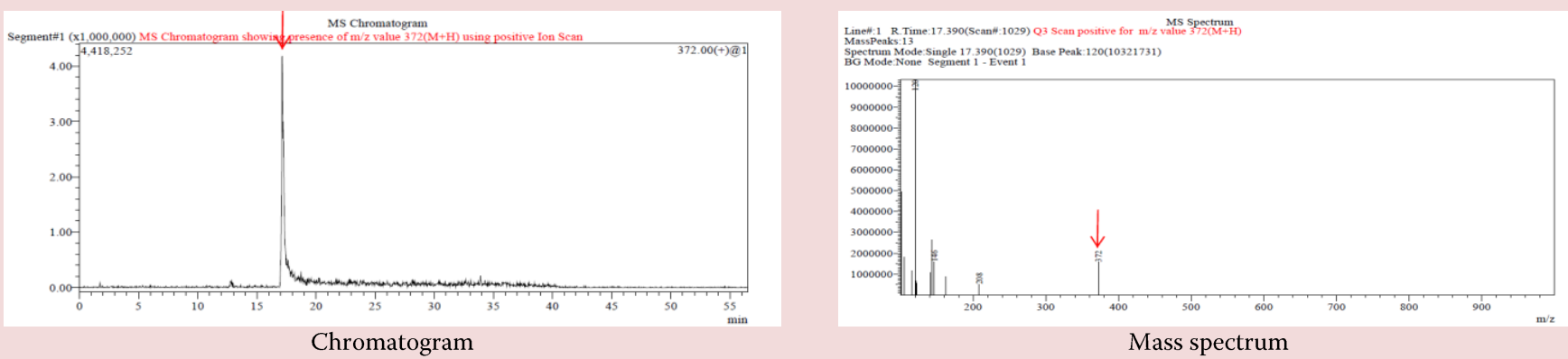

8.1 Nyagrodha
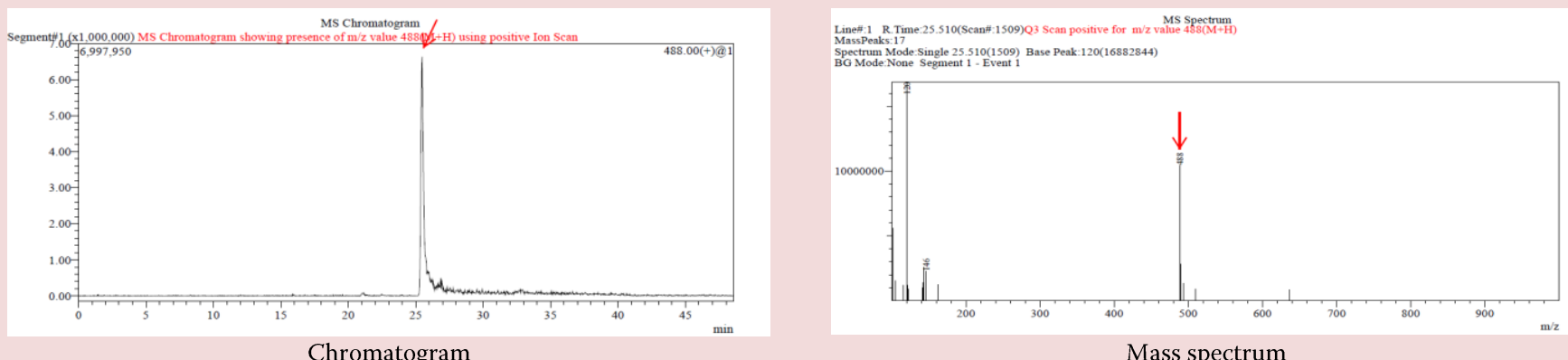

8.2 Udumbara
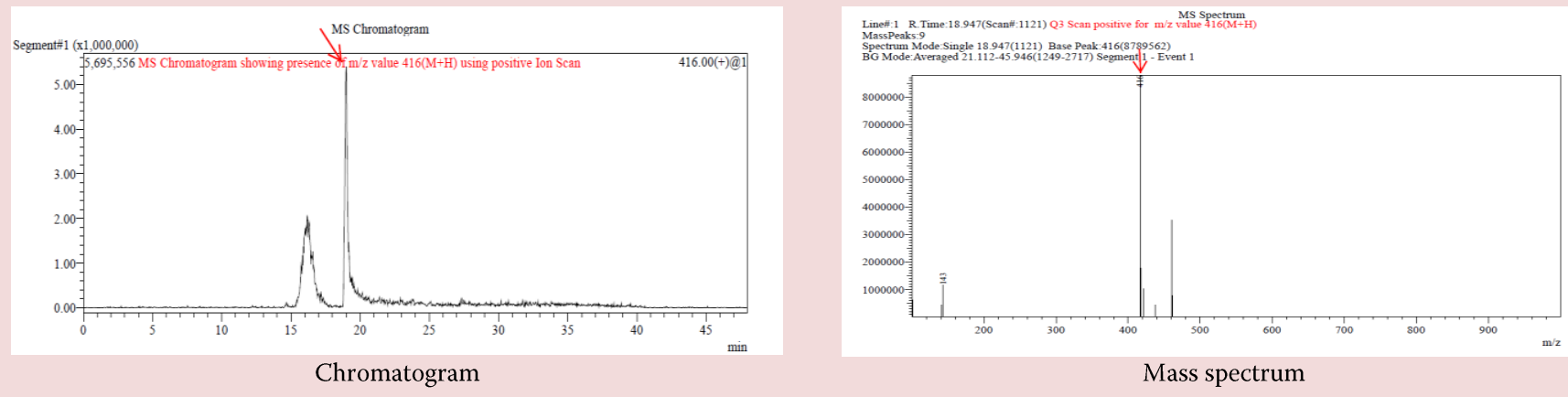

8.3 Ashwattha
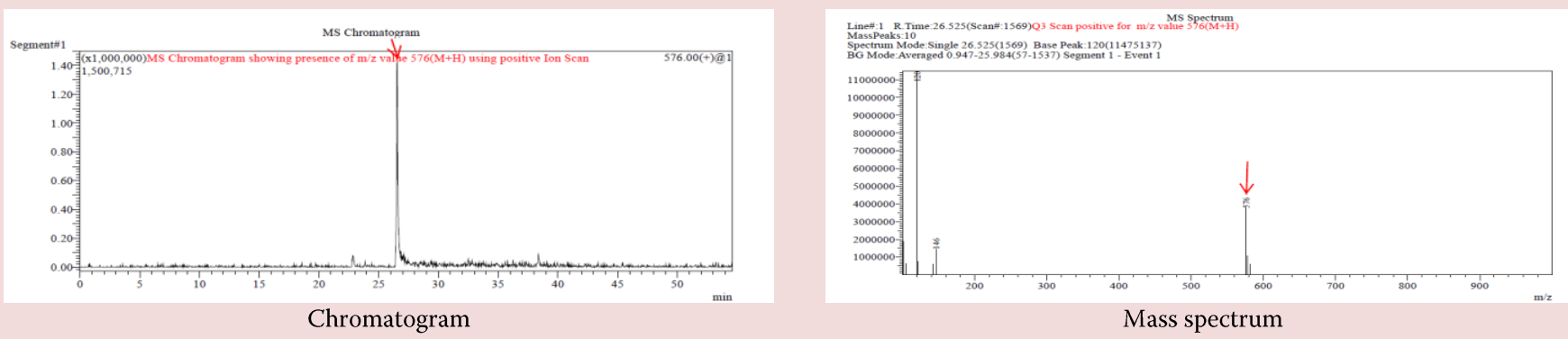

8.4 Parisa 


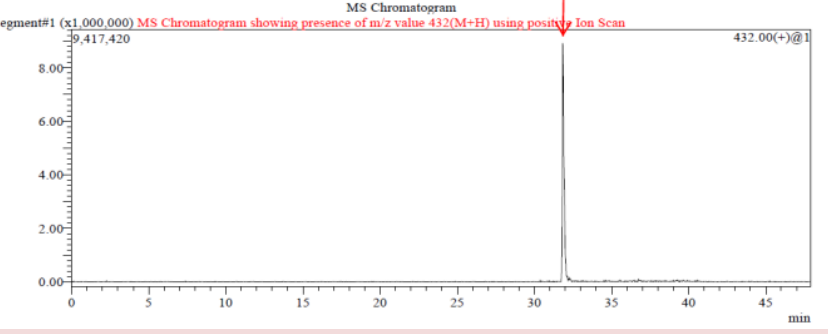

Chromatogram

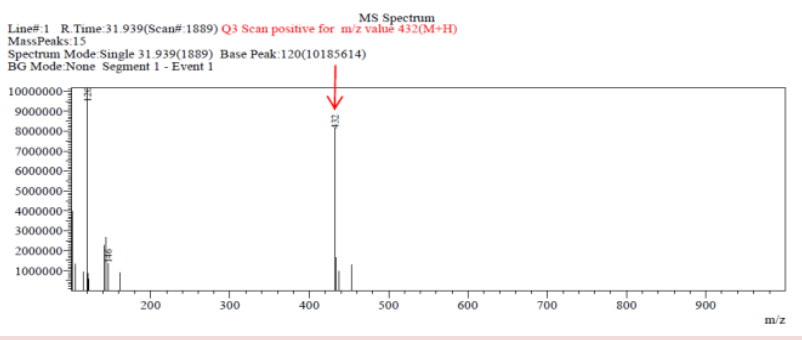

Mass spectrum

8.5 Plaksha

\section{Figure 9. LCMS Fingerprint of methanolic extract of Panchavalakala Kvatha Curnashowing peaks from its ingredients}

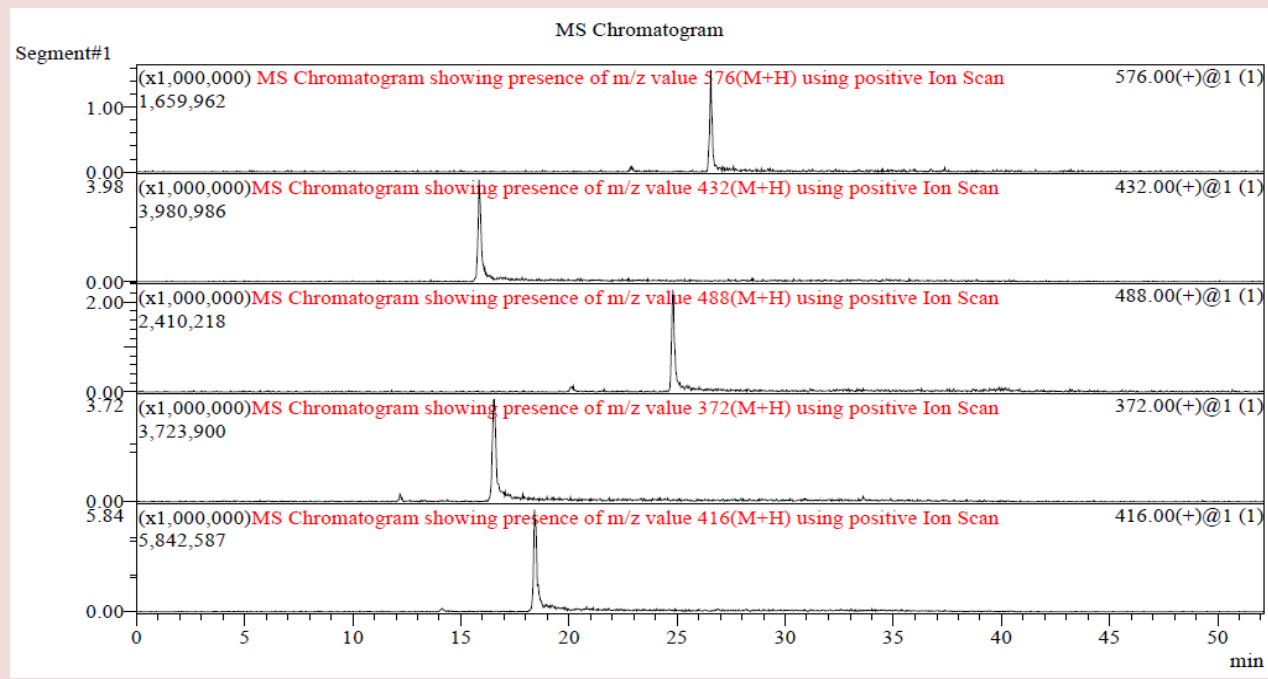

9.1 PKC

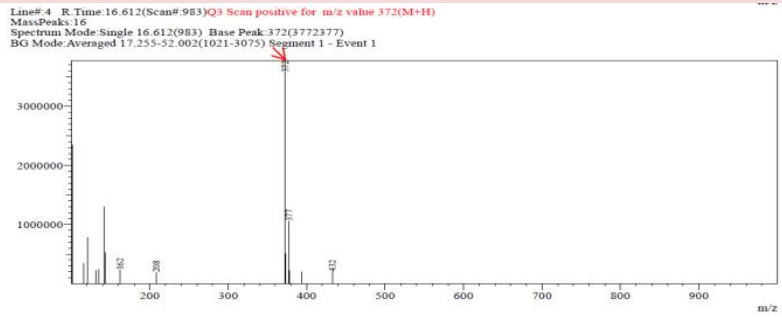

9.2 Nyagrodha

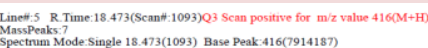

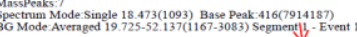

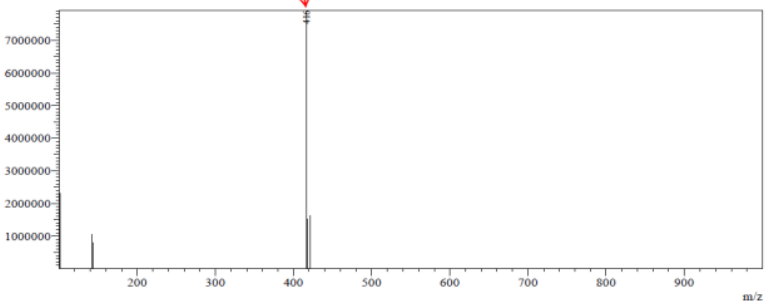

9.4 Ashwattha

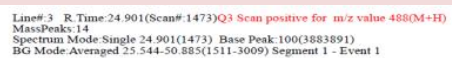

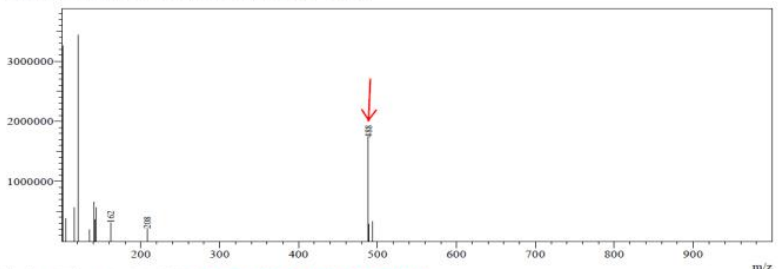

9.3 Udumbara

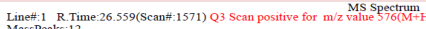

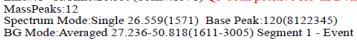

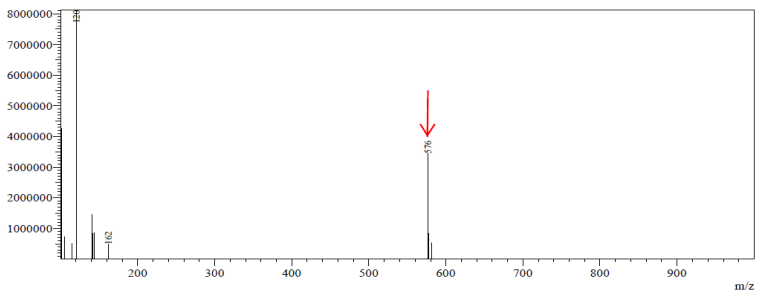

9.5 Parisa

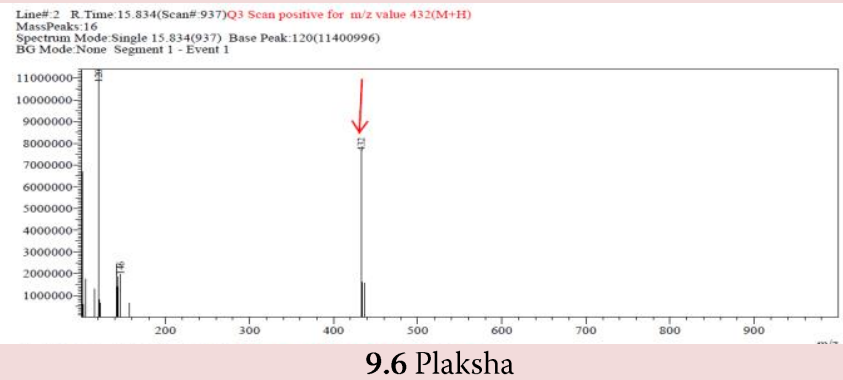


PKC has not been subjected to pharmacopoeial standards in Ayurvedic Pharmacopoeia of India so far. There is no monograph on quality standards of PKC though the formulation is very widely prescribed in Ayurvedic practice. The texture of the PKC was reported to be coarse; colour yellowish brown, with bitter taste and characteristic odour due to the specific properties of a variety of ingredients. Detailed macro-microscopic examination for identification of the ingredients and PKC will help in identifying adulterants/ substituent from the official drug along with these chemical tests $^{[11]}$.

The quality indicating fingerprints so obtained from the present study can be used as a monograph for standardization of PKC for academic platform and Ayurvedic drug manufacturing industry. The results obtained will serve as reference material for Pharmacopoeial works in the days to come.

\section{CONCLUSION}

A fact-sheet on standards for quality of Panchavalkala kvātha cūrna of Ayurvedic Formulary of India ${ }^{[12]}$ has been proposed as below:

Definition Bhūnimbādi Kvātha Cūrṇa is a coarse powder preparation made with the ingredients as per AFI formulation composition $^{[3]}$ having one part each of stem bark of Nyagrodha (Ficus benghalensis L.), Udumbara (Ficus racemosa L.), Asvattha (Ficus religiosa L.), Parisa (Thespesia populnea (L.) Sol. ex Corrêa) and Plaksha (Ficus lacor Buch.-Ham.).

Method of preparation All the ingredients of Pharmacopoeial quality were washed properly. Dried raw drugs of were coarsely powdered separately. The individual powders were passed separately through sieve number $10(1700 \mu \mathrm{m}$ IS Sieve). Each ingredient was weighed separately and mixed together in equal proportions. Passed through sieve number 10 to obtain a homogenous blend. Packed it air-tight container and stored away from direct sunlight.

Characteristics and preservation Kvātha Cūrṇa retains potency for two year and should be kept in an air tight container. Kvātha Cūrna can be used for preparing Kașāya, Hima, Phānta, etc ${ }^{[3]}$.

Physico-chemical Parameters Loss on drying at $105^{\circ} \mathrm{C}$ - Not more than 7.68 \%; Total ash - Not more than $9.83 \%$; Acidinsoluble ash - Not more than $0.60 \%$; Alcohol -soluble extractive - Not less than $15.95 \%$; Water - soluble extractive -
Not less than $7.44 \%$; pH (10\% aqueous solution) - Not more than 6.00 .

\section{Thin Layer Chromatography}

Ethanolic extract (Toluene: Ethyl Acetate: Formic Acid (5:5:0.2): Under short UV 3 bands at $R_{f} \quad 0.64,0.84$ and 0.95 (light green); under long UV 9 bands with $\mathrm{R}_{\mathrm{f}} 0.08$ (florescent light blue), 0.14 and 0.28 (fluorescent blue), 0.36, (fluorescent aqua), 0.54 (fluorescent blue), 0.66 (fluorescent light blue), 0.78 (fluorescent blue), 0.90 (fluorescent aqua) and 0.98 (fluorescent red); and after derivatisation with vanillin-sulphuric acid 7 bands with $\mathrm{R}_{\mathrm{f}} 0.06,0.35,0.8,0.90$ and 0.99 (violet).

Hydro-alcoholic extract (Toluene: Ethyl Acetate: Acetic acid: Water (3:3:0.8:0.2): Under short UV showed 4 bands at $R_{\mathrm{f}} 0.7$, 0.67, 0.71 and 0.80 (light green); under long UV showed 11 bands at $\mathrm{R}_{\mathrm{f}} 0.05,0.15,0.21,0.30,0.34,0.48,0.69,0.71,0.77,0.84$ and 0.93 (fluorescent light violet); and after derivatisation with vanillin-sulphuric acid and observation under white light 6 bands at $R_{f} 0.07,0.27,0.82,0.84,0.87,0.93$ (light violet) respectively.

Important therapeutic uses Inflammation due to wound, erysepales, syphilis/soft chancre, irrigation of wound/ulcer, leucorrhoea, conjunctivitis ${ }^{[12]}$.

Dose $48 \mathrm{~g}$ twice a day in divided dose $\mathrm{e}^{[3]}$.

Sahapana For external application after grinding with ghee or as decoction for external use ${ }^{[3]}$.

ACKNOWLEDGEMENT This research was done under major research project entitled "Standard Operating Procedures (SOPs) and Quality standards for four classical Kvātha Cūrṇas (Ayurvedic polyherbal coarse powders)" (No. RGU:R\&D:Res.Wing:2014-15 dtd. 13 MAR 2015) sanctioned by Rajiv Gandhi University of Health Sciences, Bangalore. Authors are grateful to Dr. D. Veerendra Heggade, President, SDME Society, Ujire for the support. Guidance from Dr. R. Muralidhar, Senior Manager, SDM Ayurveda Pharmacy is duly acknowledged.

\section{CONFLICT OF INTEREST Nil}

CONTRIBUTORS Dr. KN Sunil Kumar (Principal investigator) contributed to the conceptualization of the topic, manuscript review, analysis, design of the paper. Mr. Puneeth obtained the data by performing the experiments. Mrs. Suchitra contributed to drafting of the paper. Dr. Muralidhar 
(took over as principal investigator due to transfer of Dr KN Sunil Kumar) contributed to the conceptualization of the topic and contributed to the manuscript review, analysis, design and literature study.

\section{REFERENCES}

1. Agarwal S, Singh RH. Ayurveda Jan, Proceedings of International Congress; 2002;209-21.

2. European Agency for the Evaluation of Medicinal Products (EMEA). Guidelines on Quality of Herbal Medicinal Products/Traditional Medicinal Products. EMEA/CVMP/81400 Review. London. European Agency for the Evaluation of Medicinal Products; 2005.

3. The Ayurvedic Formulary of India (AFI). Part-III. $1^{\text {st }}$ ed. New Delhi: Ministry of Health and Family Welfare, Department of and Homoeopathy (AYUSH); 2011; p.86-7.

4. The Ayurvedic Pharmacopoeia of India (API). Part II. $1^{\text {st }}$ ed., Vol III. New Delhi: Ministry of Health and Family Welfare, Dept. of Ayurveda, Yoga, Unani, Siddha and Homoeopathy; 2010; p.34-7.

5. Pushpendra, KN Sunil Kumar, Priyadarshini, BS Holla, B Ravishankar, B Yashovarma. Simple modus operandi to bring down microbial load of herbal drugs to Pharmacopoeial limit - A study on ingredients of Hutabhugadi cürṇa. J Sci Innov Res 2014;2:1040-3.
6. The Ayurvedic Pharmacopoeia of India. Part I. $1^{\text {st }}$ ed., Vol VI. New Delhi: Ministry of Health and Family Welfare, Dept. of AYUSH; 2008; p.233-91.

7. Koppala Narayana Sunil Kumar, Priyadarshini, Basaviah Ravishankar, Betkeri Yashovarma. Quality standards for Bhūnimbādi Kvātha Cūrṇa. J Ayu Med Sci 2016;1(1):19-33. DOI: 10.5530/jams.2016.1.4 (ISSN 2456-4990)

8. Sethi PD. High Performance Thin Layer Chromatography. 1st ed., Vol. 10. New Delhi: CBS Publishers and Distributors; 1996; p.1-56.

9. Pushpendra, Koppala Narayana Sunil Kumar, Priyadarshini, Bantwal Shivarama Holla, Basaviah Ravishankar, Betkeri Yashovarma. Quality standards for Hutabhugadi curṇa (Ayurvedic Formulary of India). Journal of Traditional and Complementary Medicine 2016;6:78-88.

10. Sunil Kumar KN, Shakila R, Amerjothy S. Physicochemical evaluation, nutraceutical composition and HPLC-UV fingerprint of Helicanthus elastica (Desr.) Danser (Indian Mango Mistletoe). International Journal of Green Pharmacy 2014;8:175-9.

11. Mallya Suma V, Suchitra Prabhu, Vishwanatha U, Sunil Kumar KN. Anatomical atlas of Panchavalkala - effective healing five bark drugs in gynaecological disorders. J Ayu Herb Med 2018;4(1):6-13. 